Relations industrielles

Industrial Relations

\title{
Dubé, Annette et Daniel Mercure, Les entreprises et l'emploi : les nouvelles formes de qualification du travail
}

\section{Pierre Doray}

Volume 54, numéro 3, 1999

URI : https://id.erudit.org/iderudit/051260ar

DOI : https://doi.org/10.7202/051260ar

Aller au sommaire du numéro

Éditeur(s)

Département des relations industrielles de l'Université Laval

ISSN

0034-379X (imprimé)

1703-8138 (numérique)

Découvrir la revue

Citer ce compte rendu

Doray, P. (1999). Compte rendu de [Dubé, Annette et Daniel Mercure, Les entreprises et l'emploi : les nouvelles formes de qualification du travail]. Relations industrielles / Industrial Relations, 54(3), 620-622.

https://doi.org/10.7202/051260ar

Tous droits réservés @ C Département des relations industrielles de l'Université Laval, 1999
Ce document est protégé par la loi sur le droit d'auteur. L'utilisation des services d'Érudit (y compris la reproduction) est assujettie à sa politique d'utilisation que vous pouvez consulter en ligne.

https://apropos.erudit.org/fr/usagers/politique-dutilisation/ 
Les entreprises et l'emploi : les nouvelles formes de qualification du travail par Annette DUBÉ et Daniel MERCURE, Québec : Les Publications du Québec, 1997, 189 p., ISBN 2-551-17718-9.

Au cours des dernières années, de nombreux travaux ont été réalisé sur l'émergence de nouveaux modèles productifs. Plusieurs ont décrit les changements introduits et caractérisé ces nouveaux modèles. D'autres ont indiqué les limites de ces changements et même contesté le fait qu'il s'agisse d'un "vrai changement ". L'ouvrage de Dubé et Mercure s'inscrit d'emblée autour du débat sur le sens des changements récents par la présentation des différentes thèses exposées récemment. Au plan théorique, l'étude vise à tester le modèle de la flexibilité, selon lequel " la quête de flexibilité serait devenue la nouvelle trajectoire des entreprises. Cette quête témoignerait de la présence de modifications plus profondes en ce qui a trait au modèle de gestion du travail, telles que la " spécialisation flexible " des systèmes de production, l'intégration des fonctions dans l'entreprise, la réduction du nombre de niveaux hiérarchiques et, surtout, la reprofessionnalisation du travail. Une telle mutation serait annonciatrice d'un nouveau modèle de qualification du travail " (p. 2). Dubé et Mercure rappellent l'option de Kerr et Schuman pour qui il $y$ a requalification et reprofessionnalisation du procès de travail ainsi que d'autres travaux (Danièle Linhart et LEST) qui confortent plutôt la thèse opposée du maintien des formes classiques de division du travail.

L'originalité du présent ouvrage se trouve dans le fait qu'il cherche à qualifier les changements et à en évaluer la portée par l'utilisation d'un sondage réalisé auprès des entreprises de quatre secteurs industriels, plutôt que l'usage d'études de cas. L'étude vise explicitement à "repérer la nature et l'intensité des changements au cours d'une période de cinq ans " (p. 5). La démarche adoptée suit quatre étapes. Les premières permettent de décrire les changements dans la dynamique des entreprises en matière de stratégies économiques et de gestion de la main-d'œuvre. Les deux suivantes s'intéressent davantage aux qualifications requises pour l'exercice des tâches et à aux compétences privilégiées par l'employeur lors du recrutement.

Bien que l'analyse s'intéresse en particulier à l'organisation du travail et à l'évolution des compétences, l'étude cherche à cerner les différentes sources possibles de changements. Ainsi, le premier chapitre empirique examine la dynamique de transformation des entreprises en reprenant quatre formes de flexibilité (financière, technique ou productive, organisationnelle et numérique, i.e. la flexibilité dans la modulation du nombre de salariés dans l'établissement) et différentes modalités de mobilisation de la main-d'œuvre. Les auteurs notent que les entreprises sont effectivement en quête d'une plus grande flexibilité. Les changements sont notables dans de nombreuses entreprises avec des modulations d'intensité et de nature entre secteurs, ce qui conduit à conclure à l'existence de trajectoires de changement différentes selon les secteurs.

L'analyse consiste largement à dégager des logiques de changements quant aux différentes dimensions examinées: l'organisation du travail, les qualifications requises et les critères de recrutement. Ces logiques fluctuent en fonction du niveau technologique des secteurs et la population ouvrière, soit entre les ouvriers qualifiés et les ouvriers peu qualifiés. En synthèse, trois modèles de qualification émergents sont décrits. Le premier, le modèle de qualification flexible de type professionnel-conceptuel est présent chez les travailleurs qualifiés de la pétrochimie et des pâtes et papiers. Ce modèle se retrouve dans des situations caractérisées par une technologie élevée 
et une hausse de la qualification. Le deuxième modèle, flexible de type taylorisé, est le négatif du premier. Il se retrouve dans les situations industrielles où l'organisation du travail et les qualifications ont peu évolué et où le niveau technologique est plus faible. Il est particulièrement présent dans le cas des travailleurs peu qualifiés de l'industrie de l'habillement et celle du textile. Le troisième modèle, dit flexible de type adroitanalytique, est propre aux situations mixtes, soit celles où les qualifications ont le moins évoluées et l'environnement technologique est important, soit le contraire (qualifications - et technologies +). Il rend compte des pratiques des entreprises en matière de gestion du personnel peu qualifié des pâtes et papier et du personnel qualifié de l'habillement et du textile. Finalement, les travailleurs peu qualifiés de la pétrochimie connaitraient un modèle de qualification dit semi-flexible de type adroit-analytique. Pour Dubé et Mercure, la pétrochimie, et le fait de dégager ce sous-modèle en est un indicateur, est caractérisée par une dualité des voies du changement. D'un côté, nous nous retrouvons avec un modèle de professionnalisation qui se manifeste par les changements dans l'organisation du travail, les qualifications requises, l'usage des critères formels de recrutement, et de l'autre, on est plutôt devant une absence de changements. Nous pouvons nous demander si cette dualité ne tient pas à la diversité interne au secteur qui regroupe à la fois la grande entreprise pétrochimique et de petites entreprises de fabrication plastique. Les premières approfondiraient la flexibilité professionnelle et les secondes seraient organisées autour d'un modèle de qualification semi-flexible.

Ainsi, l'étude souligne que l'introduction de la flexibilité dans l'économie manufacturière n'est pas un phénomène univoque, bien que les différentes voies visent à répondre au même impératif d'introduction de flexibilité au sein des entreprises. Cela rejoint plusieurs autres travaux qui soulignent que la " modemisation " industrielle se réalise par des voies plurielles. À cet égard, les auteurs auraient gagné à confronter leurs analyses avec d'autres contributions qui ont cherché à décrire les différentes voies récentes de changement et à mieux préciser le concept de flexibilité. D'ailleurs, d'autres travaux sur les changements de paradigme productif réserve le terme de flexibilité à certaines voies de changement particulière. Lipietz, par exemple, dans Les rapports capital-travail à l'aube du XXI siècle (Paris, CEPREMAP, 1990, note $n^{\circ} 9016$ ) distingue la voie de l'implication négociée qui joue sur une requalification du travail et un renforcement de certaines règles de fonctionnement interne aux entreprises, de la flexibilité libérale qui consiste à réduire le nombre de ces règles. Flexibilité rime ici avec déréglementation. Pour Bélanger et Lévesque (" Modernisation sociale des entreprises : diversité des configurations et modèle québécois " dans La modernisation sociale des entreprises, Montréal, PUM, 1994), la flexibilité est une caractéristique de certains paradigmes et non de tous. Il s'agit du fordisme renouvelé et du modèle californien.

Malgré son intérêt manifeste, l'étude soulève plusieurs questions. La première est liée à la cohérence de l'ensemble. Le chapitre deux sur l'évolution du profil de la main-d'œuvre n'est pas réellement intégré aux autres chapitres. En soi, le chapitre est intéressant, indiquant la montée de la scolarisation de la population active et les écarts existant entre les secteurs, mais les auteurs n'y reviennent pas dans l'analyse de l'enquête ou en conclusion.

Une deuxième question est relative au choix des secteurs étudiés. Nous pourrions, bien sûr, discuter longtemps des critères pertinents de choix des secteurs et jauger l'importance relative de chacun d'eux. Pour leur part, les auteurs indiquent que les quatre secteurs choisis représentent quatre situations typiques selon la nature du procédé de production 
(petite/grande séries, usage de la maind'œuvre et niveau de technologie utilisée). À cet effet, il nous semble que la fabrication de la pâte comme du papier sont des procédés à flux continu comme la pétrochimie alors que l'industrie plastique est une industrie de grande série, ce qui la rapprocherait de l'industrie textile. Par ailleurs, certains secteurs, tout aussi stratégiques dans l'économie québécoise et par rapport à la transformation des modèles productifs, sont absents de l'échantillon. Nous pensons, par exemple, à des secteurs plus directement identifiés à la nouvelle économie comme l'industrie aéronautique ou l'industrie électronique.

La troisième question est plutôt d'ordre méthodologique. L'opérationnalisation est à plusieurs égards intéressante. Les auteurs ont utilisé plusieurs indicateurs simples qui permettaient de produire un portrait complet des changements possibles dans les différentes dimensions constitutives de l'entreprise. Par contre, il faut noter que comme dans beaucoup d'enquêtes quantitatives réalisées auprès des entreprises, le taux de réponses est faible. Il l'est particulièrement dans certains secteurs $(16,7 \%$ dans les entreprises des matières plastiques et des résines synthétiques, soit quatre entreprises sur vingt-quatre). Le nombre d'entreprises est aussi très faible dans certains secteurs (deux entreprises de la pétrochimie ont répondu). Ces deux facteurs font que les pourcentages et les indices sont, en fait, calculés sur de petits nombres. Cela soulève un problème: celuj du degré de signification des différences notées sur les différents indices entre les secteurs. Or, aucune discussion méthodologique sur cette question n'est présentée dans le texte. Cet aspect, en apparence technique, est quand mème un élément important dans la mesure où il permet de recueillir l'adhésion du lecteur quant au propos général. À cet égard, le manque d'informations techniques a créé à la lecture un malaise persistant ou un doute sur la pertinence des conclusions (l'usage du terme de malaise ou de doute est justifié parce que les auteurs ne fournissent pas les informations minimales sur la construction des indices, soit les modalités de fabrication des indices et les seuils de signification. Il apparaît alors difficile de vraiment juger de leur pertinence). Ce malaise est particulièrement fort quand on compare l'intensité des changements. En effet, en sachant que le déplacement de deux entreprises de la pétrochimie d'une catégorie de réponse à l'autre ferait modifier du tiers le pourcentage, la mesure de comparaison devient particulièrement fragile.

\section{PIERRE DORAY \\ Université du Québec à Montréal}

\section{Challenges for Work and Family in the Twenty-First Century}

edited by Dana VANNOY and Paula J. DUBECK, New York: Aldine De Gruyter, 1998, 234 p., ISBN 0-202-30567-8 (alk. paper) and 0-202-30568-6 (pbk: alk. paper).

This book stems from a November 1996 conference at the Kunz Center for the Study of Work and Family in Cincinnati, Ohio entitled "Agenda for the $21^{\text {st }}$ Century Labor Force". The fourteen chapters are grouped into four themes.

Part 2, Present Realities: Setting the Stage, provides an overview of the current dialogue about the family, work, and their interdependence. In Chapter 2 ,
Kathleen Gerson (New York University) argues that workplace resistance to changing family and gender realities as well as the conservation of structures that assume a "traditional family" create difficult dilemmas for both men and women and for which any solution requires fundamental changes in workplace structures. In Chapter 3, Michael Wallace (Indiana University) considers the implications of changes such as 